\title{
Relationship between adherence to Dietary Approaches to Stop Hypertension (DASH) diet indices and incidence of depression during up to 8 years of follow-up
}

\author{
Aurora Perez-Cornago 1, Almudena Sanchez-Villegas 2,3, Maira Bes-Rastrollo 1,3, \\ Alfredo $\mathrm{Gea}^{1,3}$, Patricio Molero ${ }^{4}$, Francisca Lahortiga-Ramos ${ }^{4}$ and \\ Miguel Ángel Martinez-Gonzalez 1,3,* \\ 'Department of Preventive Medicine and Public Health, University of Navarra, C/Irunlarrea 1, 31008 Pamplona, \\ Spain: ${ }^{2}$ Department of Clinical Sciences, University of Las Palmas de Gran Canaria, Las Palmas de Gran Canaria, \\ Spain: ${ }^{3}$ Biomedical Research Center Network on Obesity and Nutrition (CIBERobn), Physiopathology of Obesity and \\ Nutrition, Institute of Health Carlos III, Madrid, Spain: ${ }^{4}$ Department of Psychiatry and Medical Psychology, University \\ Clinic of Navarra, Pamplona, Spain
}

Submitted 7 February 2016: Final revision received 15 April 2016: Accepted 13 May 2016: First published online 23 June 2017

\begin{abstract}
Objective: Our aim was to evaluate the relationship between adherence to different Dietary Approaches to Stop Hypertension (DASH) diet indices and the risk of depression.

Design: In a prospective study we assessed 14051 participants of a dynamic (permanently ongoing recruitment) prospective cohort (the Seguimiento Universidad de Navarra (SUN) Project), initially free of depression. At baseline, a validated FFQ was used to assess adherence to four previously proposed DASH indices (Dixon, Mellen, Fung and Günther). To define the outcome we applied two definitions of depression: a less conservative definition including only self-reported physician-diagnosed depression (410 incident cases) and a more conservative definition that required both clinical diagnosis of depression and use of antidepressants (113 incident cases). Cox regression and restricted cubic splines analyses were performed.

Results: After a median follow-up period of 8 years, the multiple-adjusted model showed an inverse association with the Fung DASH score (hazard ratio $(\mathrm{HR})=0.76 ; 95 \%$ CI $0.61,0.94)$ when we used the less conservative definition of depression, and also under the more conservative definition ( $\mathrm{HR}=0.63 ; 95 \%$ CI $0.41,0.95)$. We observed a weak inverse association with the Mellen DASH score, but no statistically significant association was found for the other definitions. The restricted cubic splines analyses suggested that these associations were nonlinear (U-shaped).

Conclusions: Moderate adherence to the DASH diet as operationalized by Fung and Mellen was related to lower depression risk. Since these associations were non-linear, additional prospective studies are required before the results can be generalized and clinical recommendations can be given.
\end{abstract}

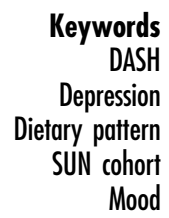

The burden of depression is on the rise and it is predicted to be one of the main causes of disability-adjusted life years by 2020 worldwide ${ }^{(1)}$. Hence, the identification of preventive dietary factors for depression has become a public health priority, as it may contribute to provide prevention opportunities to stop this alarming increase ${ }^{(2)}$.

The impact of diet on depression has received considerable attention over the past 5 years ${ }^{(2,3)}$. In this sense, the intake of single dietary nutrients such as B vitamins, folate, zinc, magnesium or long-chain $n$ - 3 fatty acids has been reported to be inversely related to the risk of depression $^{(2)}$. However, it should be acknowledged that nutrients may interact with each other and thus it might be difficult to attribute an effect to a specific nutrient or food $^{(4)}$. Therefore, research examining the role of whole dietary patterns on depression has emerged as an approach better suited to identify the association between dietary habits and depression. In this regard, healthy dietary patterns such as the Mediterranean dietary pattern have been shown to decrease the risk of depressive 
symptoms and depression diagnosis, while Westerntype diets have been proposed to do the opposite ${ }^{(2,5)}$. Moreover, it is likely that better adherence to other known healthy overall dietary patterns, including high intakes of fruit, vegetables, fish, low-fat dairy and whole grains, but low intakes of meat/meat products, saturated fat, sugar and sodium, may also contribute to lower the risk of depression $^{(2)}$.

There is a growing body of evidence suggesting an association between depression and hypertension ${ }^{(6)}$. Unhealthy lifestyle habits, like the intake of hyperenergetic and poor diets, smoking and alcohol consumption and lack of physical activity, are risk factors for both depression and hypertension. Moreover, depression and high blood pressure are associated with dysregulated redox activity signalling and elevated immuno-inflammatory response $^{(3,7)}$. These disorders may produce hyperactivation of the hypothalamic-pituitary-adrenal axis, which results in an increased secretion of corticotrophinreleasing factor, adrenocorticotrophic hormone and cortisol, known risk factors for the elevation of both blood pressure and depressive symptomatology ${ }^{(8)}$. Oxidative stress and low-grade inflammation may also cause dysfunction of the autonomic nervous system, including both increased sympathetic and decreased parasympathetic activities, promoting hypertension and depression ${ }^{(9,10)}$. Moreover, endothelial dysfunction has been proposed as a risk factor for depression due to the role of endothelial cells in the production of brain-derived neurotrophic factor. Endothelial dysfunction is also a risk factor for hypertension since it may contribute to the elevation of blood pressure and because the vascular endothelium may acquire pro-inflammatory properties ${ }^{(11)}$.

The Dietary Approaches to Stop Hypertension (DASH) eating plan, consisting of following a diet rich in fruits, vegetables and low-fat dairy foods and low in SFA and red meats, has proved to be effective in reducing inflammatory markers and cardiovascular risk factors, including blood pressure and blood lipids ${ }^{(12)}$. Also, inverse associations between adherence to the DASH diet and diabetes or CVD have been reported ${ }^{(12)}$. Since both hypertension and depression share numerous risk factors, and due to the potential benefits of the DASH diet on diabetes, CVD and low-grade inflammation, it is of interest to determine whether the dietary treatment for hypertension may be associated with depression risk. In this sense, the Mediterranean dietary pattern has been associated with reduced prevalence and incidence of both hypertension and depressive symptoms ${ }^{(2,13)}$. However, epidemiological data on the association between adherence to the DASH diet and the development of depression have not been reported yet. Therefore, the aim of the present study was to evaluate the relationship between four previously published DASH diet indices and the risk of depression in a prospective cohort of Spanish university graduates.

\section{Methods}

\section{Participants}

The 'Seguimiento Universidad de Navarra' (SUN) Project is a prospective dynamic cohort composed of alumni of the University of Navarra, registered professionals from some Spanish provinces and other university graduates ${ }^{(14)}$.

The recruitment of participants started in December 1999 and is permanently ongoing as this is a dynamic cohort study (see online supplementary material, Supplemental Fig. 1). The baseline questionnaire collected information about lifestyle factors (including a detailed FFQ) and sociodemographic and clinical variables ${ }^{(15)}$. Information with regard to exposure and outcome is collected through self-administered questionnaires sent by mail or responded to via the Internet every 2 years. Up to December 2011 , 21187 individuals were recruited. Of these, 19270 were successfully followed up (at least one follow-up questionnaire), achieving an in-person retention rate of $90 \cdot 95 \%$.

Participants who reported energy intakes outside predefined limits $(<3347 \mathrm{~kJ} / \mathrm{d}(<800 \mathrm{kcal} / \mathrm{d})$ or $>16736 \mathrm{~kJ} / \mathrm{d}$ $(>4000 \mathrm{kcal} / \mathrm{d})$ for men and $<2092 \mathrm{~kJ} / \mathrm{d}(<500 \mathrm{kcal} / \mathrm{d})$ or $>14644 \mathrm{~kJ} / \mathrm{d}$ (>3500 kcal/d) for women) were excluded from analyses ( $n$ 1807). Participants with cancer, diabetes or CVD at the beginning of the study were also excluded ( $n$ 1214), as were those who reported physician-diagnosed depression and users of antidepressant medication at baseline ( $n$ 1822). Participants with missing data on any covariate ( $n$ 40) were also excluded. Finally, data from 14051 participants were used in the statistical analyses.

The Institutional Review Board of the University of Navarra approved this study. Informed consent was implied by the voluntary completion of the first questionnaire.

\section{Exposure assessment}

The dietary exposure was determined at baseline by using a self-administered 136-item semi-quantitative FFQ, which has previously been validated in Spain ${ }^{(15)}$. Each item in the FFQ included a typical portion size. Daily food consumption was calculated by multiplying the portion size by the consumption frequency for each food item (nine options ranging from never or almost never to six or more times per day). The nutrient composition of the dietary intake items was derived from Spanish food composition tables $^{(15)}$.

We tested four previously published scores, namely those of Dixon, Mellen, Fung and Günter, to operationally assess adherence to the DASH dietary pattern. Four different DASH diet indices were analysed since a previous study found that differences in how the DASH dietary pattern was operationalized affected the ability of the DASH indices to predict disease risk ${ }^{(16)}$. Briefly, these indices give higher values to adequate adherence and lower to inadequate adherence, giving slightly different scores to the food groups or nutrients included in each of the four scores. Dixon's DASH index is composed of eight 
food groups and one nutrient component, and it ranges from 0 to 8 . Mellen's DASH index is comprised of nine nutrients and its total scores ranges from 0 to 9. Fung's DASH index is composed of eight components (seven food groups and one nutrient) which are subsequently divided into quintiles, and gives higher points to the fifth quintile (higher consumption) for the healthy items and higher points to the first quintile (lower consumption) for the unhealthy items; the total score ranges from a minimum of 8 to a maximum of 40 points. Finally, Günther's DASH index is composed by ten components, given a maximum score of 10 when the recommendations are achieved, while lower intakes are scored proportionately. The total score ranges from 0 to 80. Detailed information about how these DASH diet indices were calculated can be found in the online supplementary material, Supplemental Table 1.

Supplemental Table 2 (see online supplementary material) shows the pairwise correlation matrix between each DASH diet index. Further information on their composition and how to calculate these DASH diet indices can be found elsewhere ${ }^{(17-20)}$.

\section{Outcome assessment}

The follow-up questionnaires were used to obtain the outcome (4, 6 and 8 years of follow-up; respectively Q_4, Q_6 and Q_8; see online supplementary material, Supplemental Fig. 1). If incident depression was missing in any of the follow-up questionnaires (Q_4, Q_6 or Q_8) we used the participants' answers to a brief questionnaire (a shorter version of the follow-up questionnaires used only when participants, after several reminders, do not answer to fulllength follow-up questionnaires). Participants reporting depression at baseline as well as early cases of depression (physician-diagnosis or antidepressant use reported within the first 2 years of follow-up) were excluded from the analysis to avoid reverse causation bias. Two definitions of Major Depressive Disorder, both of them self-reported, were used: (i) a less conservative (broad) definition that included a positive answer to the question 'Have you ever been diagnosed of depression by a medical doctor?'; and (ii) a more conservative (strict) definition that required both self-reported physician-diagnosed depression and use of antidepressant medication by participants in any of the follow-up questionnaires (Q_4, Q_6 or Q_8, or the short questionnaire). A psychiatrist used a sub-sample of our cohort using the Structured Clinical Interview for DSM-IV (SCID-I) as a gold standard in order to validate the broader definition of incident depression (first definition). The percentage of confirmed cases of depression was 74.2 (95\% CI $63.3,85 \cdot 1) \%$, while the percentage of confirmed cases of non-depression was $81 \cdot 1$ (95\% CI 69.1, 92.9) \% ${ }^{(21)}$.

\section{Covariate assessment}

The baseline questionnaire was used to obtain most of the covariates. Information regarding sociodemographic characteristics (age, sex, marital and employment status), anthropometric (weight, height and BMI) variables, and lifestyle and health-related habits (smoking status and leisure-time physical activity) were collected. In the baseline questionnaire participants also answered questions about personality and behaviour features using Likert-type scales with values in the range $0-10$, including the level of competitiveness (higher scores, more competitive), level of anxiety (higher scores, more anxious) and level of dependence (higher scores, more dependant). Body weight was collected in the follow-up questionnaires (every 2 years) and the 'weight change' variable was created by resting the body weight until depression incidence appears or end to followup to the weight at baseline (for example, body weight in the second year of follow-up - body weight at baseline). Physical activity was evaluated using a validated questionnaire $^{(5,22)}$. Moreover, the prevalence of hypertension at baseline (self-reported) was also ascertained, to assess potential interactions, since the DASH diet was specifically designed to combat hypertension and can be especially suited for hypertensive patients.

\section{Statistical analysis}

Baseline characteristics of participants stratified by the higher and lower categories of each of the four DASH diet indices were described using relative frequencies, means and standard deviations. The four indices were divided into quintiles except for Dixon's DASH index, which was divided into four categories due to its low resolution. The $\chi^{2}$ test for trend (categorical variables) and ordinary leastsquares linear regression analysis (continuous variables) across quintiles of DASH diet indices were used for the comparisons of baseline characteristics.

Time-to-event methods with Cox regression models were used to assess the relationship between the different categories of adherence to the DASH diet indices and the incidence of depression during follow-up. Each of the four DASH diet indices was divided into quintiles except for the Dixon's DASH index which was divided into quartiles due the small number of participants in the extreme categories. Hazard ratios (HR) and their 95\% confidence intervals were calculated using the lowest quintile or quartile of each DASH diet index as the reference category. The Cox model included age at baseline (when the FFQ was completed) as the entry time variable. Birth date was used as the origin variable (i.e. age was the underlying time variable in Cox models). Exit time was defined as the date when completing the last follow-up questionnaire, death, or their date of diagnosis for participants who developed depression. Analyses were stratified by date of recruitment (2-year periods) and deciles of age. To check for proportional hazard assumption, we used time-varying covariates and the Schoenfeld residuals.

In all analyses, we applied multiple-adjusted models where the following potential confounders were included as covariates: age, sex, smoking (never, current, former), physical activity (quartiles), total energy intake (kcal/d), 
living alone (yes/no), marital status (married or not), employment status (unemployed or no), baseline hypertension (yes/no), weight change (until depression incidence appears or end to follow-up, quartiles), and the personality traits of competitiveness (higher scores, more competitive), relaxation/stress (lower scores, more relaxed) and dependency (higher scores, more dependant) ranging from 0 to 10. We evaluated the interaction between each of the four DASH diet indices and baseline hypertension, age categories (deciles of age) and sex by introducing interaction terms in separate models, and then comparing the models with and without the interaction term with likelihood ratio tests. Tests of linear trend across increasing categories of adherence to the DASH diet were conducted by assigning medians to each category and treating this variable as continuous in the Cox regression model. Moreover, Pearson's correlation coefficients were calculated to identify the relationship between each DASH diet index.

Finally, the possible non-linear relationship between adherence to the DASH diet indices and incident depression was further examined with restricted cubic splines analyses with four knots. To test for non-linearity, we used the likelihood ratio test, comparing the model with only the linear term and the model with the linear and the cubic spline terms. The models were adjusted for the same potential confounders as the main Cox regression analyses (previously explained). All $P$ values were two-tailed and $P<0.05$ was considered significant. Analyses were performed using the statistical software package STATA/SE version 12.1.

\section{Results}

The main characteristics of the 14051 participants categorized according to their adherence to the different DASH diet indices are presented in Table 1. Participants with higher adherence to the four DASH diet indices were more likely to be married, women and physically more active. According to the less conservative definition of depression, after a median follow-up of 8 years, a total of 410 new cases of depression during the follow-up period were identified, which accrued 100434 person-years, while for the more conservative definition 113 new cases were documented (83627 person-years).

The association between baseline adherence to the DASH diet indices and the risk of depression considering the less conservative definition of depression is presented in Table 2. The Cox regression analysis using baseline adherence to the DASH diet indices as exposure showed a positive association between adherence to the Dixon index of the DASH diet and the incidence of depression (highest $v$. lowest category of adherence: $\mathrm{HR}=1.44$; $95 \%$ CI 1.05, 1.99). A non-linear, U-shaped relationship between depression risk and adherence to the Mellen, Fung and Günther DASH diet indices was found. In the multiple-adjusted models, we observed that the HR for developing depression decreased significantly in the second and third quintiles of adherence to the Mellen DASH index (HR $=0.68 ; 95 \%$ CI $0.51,0.91$ for the second quintile; $\mathrm{HR}=0.55 ; 95 \% \mathrm{CI} 0.37,0.83$ for the third quintile), in the second and fourth quintiles of adherence to the Fung DASH index ( $\mathrm{HR}=0 \cdot 62 ; 95 \% \mathrm{CI} 0 \cdot 46,0 \cdot 84$ for the second quintile; $\mathrm{HR}=0.68 ; 95 \% \mathrm{CI} 0.51,0.92$ for the fourth quintile) and in the third quintile of adherence to the Günther DASH index (HR $=0.72 ; 95 \%$ CI 0.52, 0.99). However, this association was stronger in the Fung DASH index. When the more conservative definition of depression was used (diagnosis of depression plus antidepressant use), the apparent inverse association between incidence of depression and adherence to the Mellen and Fung DASH indices was maintained (see online supplementary material, Supplemental Table 3). No statistically significant interactions between sex, age or hypertension and adherence to any of the analysed DASH diet indices were found (data not shown).

Another Cox regression analysis was conducted after merging all participants belonging to the upper categories (3-9 points or Q2-Q5) of adherence to the DASH diet indices in a single group. We compared them with those participants in the lowest category ( $\leq 2$ points or Q1) of adherence (Table 3 ). The rationale for using this cut-off point is supported by the possibility that only a clearly suboptimal nutrient intake (e.g. of some micronutrients such as magnesium, potassium and calcium, and also vitamin $\mathrm{B}_{6}$, folate and vitamin $\mathrm{B}_{12}$, which are needed for the synthesis of active bioamines) might be related to a higher risk of depression and it may be present only in the lowest category of adherence to a healthy dietary pattern, such as the DASH diet. We repeated these analyses using both the less conservative and the more conservative definitions of incident depression. No significant differences were found for the indices proposed by Dixon and Günter. However, the upper categories of adherence to the Fung DASH index exhibited lower depression risk in both definitions of depression. Moreover, a lower risk of depression was also observed in the upper categories of adherence to the Mellen DASH index only for the less conservative definition of depression.

To account for non-linear associations, models with restricted cubic spline analysis adjusted for the same potential confounding factors were used (Fig. 1). We found a non-linear association between adherence to Mellen's $(P$ for non-linearity $=0 \cdot 008)$ and Fung's $(P$ for non-linearity $=0.003)$ DASH diet indices and the incidence of depression; for Dixon's and Günter's DASH indices, $P$ for non-linearity was $>0 \cdot 100$.

\section{Discussion}

The results from the current prospective study suggest that adherence to the Mellen and the Fung DASH diet indices, 
Table 1 Baseline characteristics of the SUN study population according to extreme quintiles of DASH diet index scores; SUN Project, 1999-2011

\begin{tabular}{|c|c|c|c|c|c|c|c|c|c|c|c|c|c|c|c|c|c|c|}
\hline & & & \multicolumn{4}{|c|}{ Dixon's DASH index } & \multicolumn{4}{|c|}{ Mellen's DASH index } & \multicolumn{4}{|c|}{ Fung's DASH index } & \multicolumn{4}{|c|}{ Günther's DASH index } \\
\hline & \multicolumn{2}{|c|}{ All participants } & \multicolumn{2}{|c|}{$\leq 2$ points } & \multicolumn{2}{|c|}{$\geq 5$ points } & \multicolumn{2}{|c|}{ Quintile 1} & \multicolumn{2}{|c|}{ Quintile 5} & \multicolumn{2}{|c|}{ Quintile 1} & \multicolumn{2}{|c|}{ Quintile 5} & \multicolumn{2}{|c|}{ Quintile 1} & \multicolumn{2}{|c|}{ Quintile 5} \\
\hline & Mean & SD & Mean & SD & Mean & SD & Mean & SD & Mean & SD & Mean & SD & Mean & SD & Mean & SD & Mean & SD \\
\hline$n$ & \multicolumn{2}{|c|}{14051} & \multicolumn{2}{|c|}{4598} & \multicolumn{2}{|l|}{1752} & \multicolumn{2}{|c|}{3747} & \multicolumn{2}{|c|}{2678} & \multicolumn{2}{|l|}{3581} & \multicolumn{2}{|c|}{2372} & \multicolumn{2}{|l|}{3041} & \multicolumn{2}{|c|}{2402} \\
\hline Sex (\% female) & \multicolumn{2}{|c|}{59.1} & \multicolumn{2}{|c|}{44.8} & \multicolumn{2}{|c|}{87.9} & \multicolumn{2}{|c|}{47.5} & \multicolumn{2}{|c|}{$71 \cdot 3$} & \multicolumn{2}{|c|}{$57 \cdot 9$} & 60 & & 46 & & & 7 \\
\hline Age (years) & 37.5 & 11.5 & 38.5 & $11 \cdot 6$ & 37.4 & 11.9 & $34 \cdot \varepsilon$ & $10 \cdot 4$ & $40 \cdot 7$ & $12 \cdot 3$ & $33 \cdot 3$ & $9 \cdot 6$ & $42 \cdot 1$ & $12 \cdot 4$ & 35.4 & $10 \cdot 8$ & 39.5 & $12 \cdot 3$ \\
\hline BMI $\left(\mathrm{kg} / \mathrm{m}^{2}\right)$ & 23.5 & 3.4 & 24.0 & 3.5 & 22.5 & 3.2 & 23. & 3.4 & 23.4 & 3.4 & $23 \cdot 0$ & 3.3 & 23.5 & 3.3 & $23 \cdot 6$ & 3.5 & $23 \cdot 0$ & $3 \cdot 1$ \\
\hline $\begin{array}{l}\text { Leisure-time physical activity } \\
(\mathrm{MET} \times \mathrm{h} / \text { week })\end{array}$ & $21 \cdot 7$ & 23.0 & $20 \cdot 1$ & $20 \cdot 4$ & $25 \cdot 4$ & $27 \cdot \overline{6}$ & 19. & $20 \cdot 5$ & $25 \cdot 2$ & $27 \cdot 1$ & $18 \cdot 2$ & $20 \cdot 3$ & $27 \cdot 7$ & $28 \cdot 4$ & $18 \cdot 8$ & 21.5 & $26 \cdot 9$ & $27 \cdot 3$ \\
\hline Unemployed (\%) & & 0 & & 5 & 5 & & & & & & 4.9 & & & 8 & 4 & 5 & & 0 \\
\hline Smoking status (\%) & & & & & & & & & & & & & & & & & & \\
\hline Never smoker & $4 \varepsilon$ & & & & 52 & & & & & & 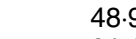 & & 49 & & 44 & & & 8 \\
\hline Former smoker & $2 \varepsilon$ & & & & 28. & & & & & & $21 \cdot 1$ & & & & 24 & & & .1 \\
\hline Current smoker & 21 & & & & 16 & & & & & & 27.4 & & & & 28 & & & 0 \\
\hline Marital status (\% married) & 50 & & & & 45 & & & & & & 39.8 & & & & 46 & & & 0 \\
\hline Living alone (\%) & & 4 & & 3 & 8 & & & & & & $5 \cdot c$ & & & 5 & 4 & & & 4 \\
\hline Total energy intake $(\mathrm{kJ} / \mathrm{d})$ & 9862 & 2573 & 9540 & 2418 & 10397 & 2586 & 10791 & 2556 & 8920 & 2469 & 10259 & 2544 & 9678 & 2389 & 10083 & 2715 & 9954 & 2385 \\
\hline Total energy intake $(\mathrm{kcal} / \mathrm{d})$ & 2357 & 615 & 2280 & 578 & 2485 & 618 & 2579 & 611 & 2132 & 596 & 2452 & 608 & 2313 & 571 & 2410 & 649 & 2379 & 570 \\
\hline Hypertension (\%) & & 8 & & 7 & 5 & & & & & & 4.0 & & & & 5 & & & 3 \\
\hline Weight change $(\mathrm{kg})$ & 1.5 & $6 \cdot 1$ & 1.5 & $6 \cdot 1$ & $1 \cdot 1$ & $6 \cdot 1$ & $1 \cdot \varepsilon$ & $6 \cdot 3$ & $1 \cdot 1$ & $6 \cdot 7$ & 1.9 & $6 \cdot 1$ & 0.9 & $5 \cdot 9$ & 1.5 & $6 \cdot 1$ & 1.5 & $6 \cdot 1$ \\
\hline Personality traits & & & & & & & & & & & & & & & & & & \\
\hline Competitive & $7 \cdot 0$ & 1.7 & $7 \cdot 0$ & 1.7 & $7 \cdot 0$ & $1 \cdot 7$ & 6. & $1 \cdot 7$ & $7 \cdot 1$ & 1.7 & $7 \cdot 0$ & 1.7 & $7 \cdot 0$ & 1.7 & 6.9 & 1.8 & $7 \cdot 0$ & 1.7 \\
\hline Relaxed & 5.9 & $2 \cdot 2$ & 5.9 & $2 \cdot 2$ & 5.9 & $2 \cdot 2$ & 5. & $2 \cdot 2$ & 5.9 & $2 \cdot 2$ & $5 \cdot 9$ & $2 \cdot 2$ & $5 \cdot 8$ & $2 \cdot 2$ & $5 \cdot 9$ & $2 \cdot 2$ & $5 \cdot 9$ & $2 \cdot 2$ \\
\hline Dependant & 3.6 & $2 \cdot 8$ & 3.6 & $2 \cdot 9$ & 3.5 & $2 \cdot 9$ & $3 \cdot($ & $2 \cdot 7$ & 3.5 & 3.0 & $3 \cdot 7$ & $2 \cdot 7$ & 3.4 & 2.9 & $3 \cdot 7$ & $2 \cdot 8$ & 3.5 & $2 \cdot 9$ \\
\hline
\end{tabular}

SUN, Seguimiento Universidad de Navarra/University of Navarra Follow-up; DASH, Dietary Approaches to Stop Hypertension; MET, metabolic equivalent of task.

Indicates $P>0.05$ for $x^{2}$ test for trend (categorical variables) and linear regression analysis (continuous variables) across quintiles of DASH diet indices. 
Table $2 \mathrm{HR}$ and $95 \% \mathrm{Cl}$ for incident depression (diagnosis of depression) according to baseline categories of adherence to the DASH diet indices in the SUN study population; SUN Project, 1999-2011

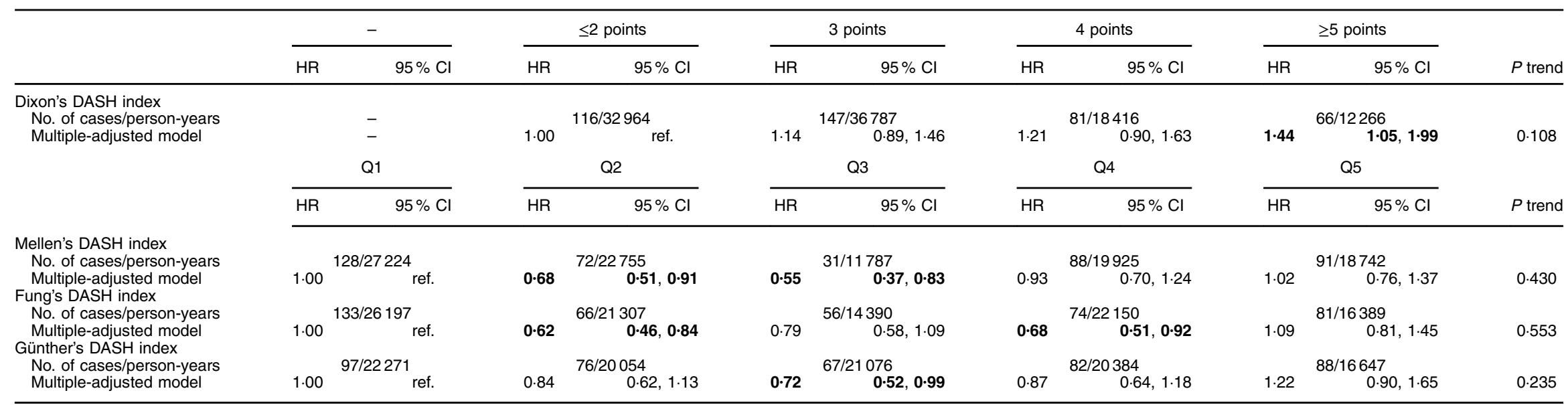

HR, hazard ratio; SUN, Seguimiento Universidad de Navarra/University of Navarra Follow-up; DASH, Dietary Approaches to Stop Hypertension; ref., reference category. HR estimated with Cox regression and $95 \% \mathrm{Cl}$. If the $\mathrm{Cl}$ includes 1.00 , the results are not significant (two-tailed $P>0.05)$. Bold values indicate statistical significance $(P<0.05)$. Multiple-adjusted model: adjusted for sex

Table $3 \mathrm{HR}$ ratios and $95 \% \mathrm{Cl}$ for incident depression according to baseline categories of adherence to the DASH diet indices in in the SUN study population; SUN Project, 1999-2011

\begin{tabular}{|c|c|c|c|c|c|c|c|c|}
\hline & \multicolumn{4}{|c|}{ Less conservative definition (diagnosis of depression) } & \multicolumn{4}{|c|}{ More conservative definition (diagnosis of depression and antidepressant use) } \\
\hline & \multicolumn{2}{|c|}{$\leq 2$ points } & \multicolumn{2}{|c|}{ 3-9 points } & \multicolumn{2}{|c|}{$\leq 2$ points } & \multicolumn{2}{|c|}{ 3-9 points } \\
\hline & $\mathrm{HR}$ & $95 \% \mathrm{Cl}$ & $\mathrm{HR}$ & $95 \% \mathrm{Cl}$ & $\mathrm{HR}$ & $95 \% \mathrm{Cl}$ & $\mathrm{HR}$ & $95 \% \mathrm{Cl}$ \\
\hline \multirow{4}{*}{$\begin{array}{l}\text { Dixon's DASH index } \\
\text { No. of cases/person-years } \\
\text { Multiple-adjusted model }\end{array}$} & \multicolumn{2}{|c|}{$119 / 32964$} & \multicolumn{2}{|c|}{$294 / 67469$} & \multicolumn{2}{|c|}{$28 / 27362$} & \multicolumn{2}{|c|}{$85 / 56265$} \\
\hline & 1.00 & ref. & $1 \cdot 21$ & $0.97,1.51$ & 1.00 & ref. & 1.47 & $0.95,2 \cdot 30$ \\
\hline & \multicolumn{2}{|c|}{ Q1 } & \multicolumn{2}{|c|}{ Q2-Q5 } & \multicolumn{2}{|c|}{ Q1 } & \multicolumn{2}{|c|}{ Q2-Q5 } \\
\hline & $\mathrm{HR}$ & $95 \% \mathrm{Cl}$ & $\mathrm{HR}$ & $95 \% \mathrm{Cl}$ & $\mathrm{HR}$ & $95 \% \mathrm{Cl}$ & $\mathrm{HR}$ & $95 \% \mathrm{Cl}$ \\
\hline \multicolumn{9}{|l|}{ Mellen's DASH index } \\
\hline No. of cases/person-years & \multicolumn{2}{|c|}{$128 / 27224$} & \multicolumn{2}{|c|}{$282 / 73209$} & \multicolumn{2}{|c|}{$37 / 22390$} & \multicolumn{2}{|c|}{$76 / 61237$} \\
\hline Multiple-adjusted model & 1.00 & ref. & 0.80 & $0.64,1.00$ & 1.00 & ref. & 0.68 & $0.45,1.04$ \\
\hline Fung's DASH index & \multirow{2}{*}{\multicolumn{2}{|c|}{$133 / 26197$}} & \multirow{2}{*}{\multicolumn{2}{|c|}{$277 / 74237$}} & \multirow{2}{*}{\multicolumn{2}{|c|}{$37 / 21595$}} & \multirow{2}{*}{\multicolumn{2}{|c|}{$76 / 62032$}} \\
\hline No. of cases/person-years & & & & & & & & \\
\hline Multiple-adjusted model & 1.00 & ref. & 0.76 & $0.61,0.94$ & 1.00 & ref. & 0.63 & $0.41,0.95$ \\
\hline Günther's DASH index & \multirow{2}{*}{\multicolumn{2}{|c|}{$97 / 22271$}} & \multirow{2}{*}{\multicolumn{2}{|c|}{$313 / 78162$}} & \multirow{2}{*}{\multicolumn{2}{|c|}{$23 / 18276$}} & \multirow{2}{*}{\multicolumn{2}{|c|}{$90 / 65351$}} \\
\hline $\begin{array}{l}\text { No. of cases/person-years } \\
\text { Multiple-adjusted model }\end{array}$ & & & & $\begin{array}{l}62 \\
0.69,1 \\
1.11\end{array}$ & & & & \\
\hline
\end{tabular}

HR, hazard ratio; SUN, Seguimiento Universidad de Navarra/University of Navarra Follow-up; DASH, Dietary Approaches to Stop Hypertension; ref., reference category.

HR estimated with Cox regression and $95 \% \mathrm{Cl}$ If the Cl includes 1.00 the results are not significant (two-tailed $P>0.05)$. Bold values indicate statistical significance ( $P<0$. 05 ). Multiple-adjusted model: adjusted for sex,

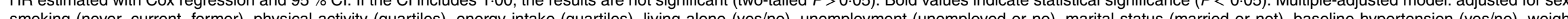
change (quartiles), and for the personality traits competitive, relaxed and dependant (from 0 to 10). All models were stratified by date of recruitment (2-year periods) and deciles of age. 

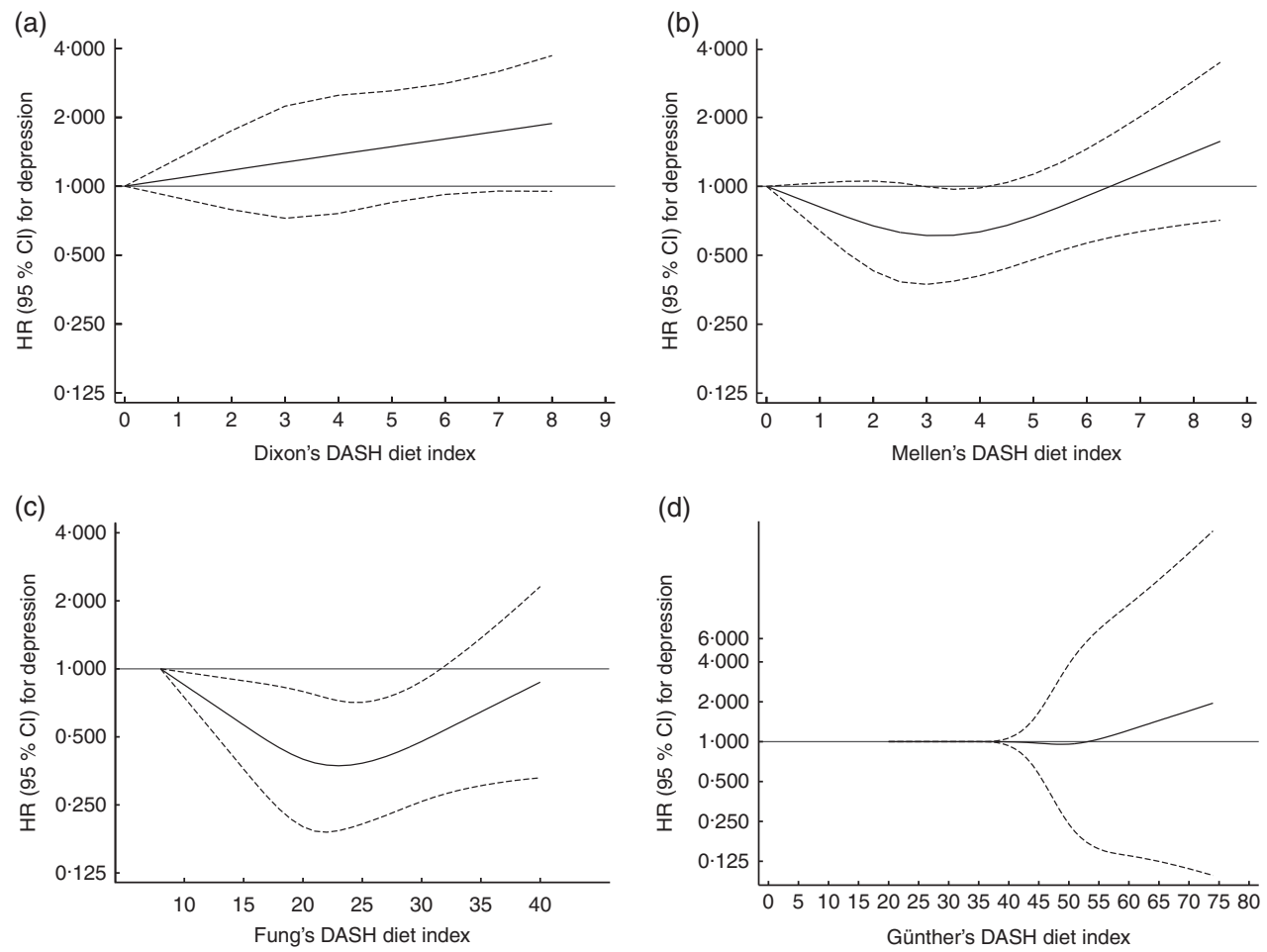

Fig. 1 Relationship between each DASH diet index (a, Dixon; b, Mellen; c, Fung; d, Günter) and depression risk in the SUN study population ( $\mathrm{HR}$ and $95 \% \mathrm{Cl}$ ) using the less conservative definition of depression (diagnosis of depression); SUN Project, 19992011. Restricted cubic spline model in which —_ represent the HR and - - - - - represent the $95 \% \mathrm{Cl}$. The multiple-adjusted model was stratified by date of recruitment (2-year periods) and deciles of age, and adjusted for sex, age, smoking (never, current, former), physical activity (quartiles), energy intake (quartiles), living alone (yes/no), unemployment (unemployed or no), marital status (married or not), baseline hypertension (yes/no) and weight change (quartiles), and for the personality traits competitive, relaxed and dependant (from 0 to 10). DASH, Dietary Approaches to Stop Hypertension; SUN, Seguimiento Universidad de Navarra/University of Navarra Follow-up; HR, hazard ratio

but especially the Fung DASH diet index, was inversely associated with depression risk. There was evidence of non-linear associations between adherence to the Mellen and the Fung DASH diet indices and risk of depression. We also found a weak positive association between the Dixon DASH index and incident depression. To our knowledge, this is the first epidemiological study that has assessed the association between adherence to four established DASH diet indices and the incidence of depression.

Only the Mellen and the Fung DASH indices showed significant inverse associations with the incidence of depression. An important difference between the four studied DASH diet indices is the scoring system. Also, some indices are composed by specific food choices, whereas others reflect nutrient intakes. While the Mellen DASH index is more focused on nutrient intake, the Fung DASH index reflects food choices ${ }^{(16)}$. Surprisingly, the Dixon DASH index was positively associated with incident depression when the index was stratified into four categories. However, this association was not significant after merging all participants belonging to the upper categories (3-9 points) in a single group and comparing them with those participants in the lowest category ( $\leq 2$ points) of adherence, nor when the more conservative definition of depression was used, nor in the spline regression model. Therefore, we suggest that the isolated significant positive association might be due to multiple testing or residual confounding, since it was not significant in the rest of our analyses.

Among the components of the Mellen and the Fung DASH diet indices there are several food groups and nutrients that have been related with lower risk of developing depression. Mellen's DASH index considers several minerals that have previously been related to lower depression risk, such as calcium and potassium ${ }^{(23)}$, and maybe these components make the difference. Specifically, magnesium, potassium and calcium have been proposed as having a protective effect against psychiatric disorders $^{(23)}$, while high sodium intake has been associated with increased urinary cortisol and its metabolites, insulin resistance and the metabolic syndrome, known risk factors for depression ${ }^{(24,25)}$. The Mellen DASH index takes fibre content into account; however, the possible involvement of fibre in mood disorders has so far not been investigated much, although a negative association has been observed ${ }^{(26)}$. On the other hand, Fung's DASH index takes food groups into account. High SFA as well as total 
dietary fat consumption has been related to higher depression risk, although this effect has not been much studied $^{(2,26)}$. Moreover, fruits and vegetables are good sources of B vitamins and antioxidants, which appear to be important in reducing depressive symptomatology ${ }^{(2,27)}$. Although legumes have not been independently associated with depression, these food groups are good sources of fibre, B vitamins and minerals, nutrients that appear to be important in reducing depressive symptoms ${ }^{(26,27)}$. Also, the possible beneficial effects of the Fung DASH diet index compared with the others may reside in that it is the only index that takes into account the consumption of sugar-sweetened beverages. High sugarsweetened beverage consumption might produce an increased hypothalamic-pituitary-adrenal axis activity ${ }^{(28)}$, which may increase cortisol levels and hence depression risk $^{(29)}$. Additionally, the degree of non-differential misclassification was lower in the Fung index than in the other indices (see online supplementary material, Supplemental Table 4), and this ability of the Fung index to better capture the actual DASH diet was a likely explanation of the stronger association found with this index.

In some of our analyses we separated the lowest category of adherence from the rest of categories. The rationale was related to exploring the possibility that only a clearly suboptimal nutrient intake (including their interactions) is related to a higher risk of depression and it may be present only in the lowest category of adherence to a healthy dietary pattern, such as the DASH diet. Once a sufficient level of nutrient intake is reached no further benefit is obtained. In this line of thought, not only magnesium, potassium and calcium, but also a widely accepted aetiological hypothesis of depression is related to the availability of serotoninergic or noradrenergic monoamines in the central nervous system and their availability in the synapse $\mathrm{e}^{(30)}$. It may be speculated that some micronutrients or a set of metabolically relevant cofactors related to the synthesis of these monoamines may present suboptimal intakes mainly in the group with the lowest adherence to the DASH pattern.

Several dietary patterns have been recently related to depression; however, most of these studies had a crosssectional design ${ }^{(2)}$, whereas also longitudinal studies have found similar results ${ }^{(5,31)}$. To follow a healthy dietary pattern has been inversely associated with depressive symptoms ${ }^{(2,32)}$. Similarly, the Mediterranean dietary pattern might prevent the development of future depression ${ }^{(5,31)}$, while unhealthy dietary habits like fast food, processed food and trans-unsaturated fat consumption were related to an increased risk of suffering depression $^{(2,32)}$. Importantly, the Mediterranean dietary pattern includes healthy fats such as olive oil and oily fish among its components; however, the DASH diet considers total fat intake a harmful component of the diet. An increased consumption of oily fish and olive oil has been related with reduced depressive symptoms ${ }^{(33)}$. This may explain why a clear inverse association between the Mediterranean dietary pattern and depression was previously found in this cohort ${ }^{(5)}$, but not a very strong association between the DASH diet and incidence of the depression has been detected in the present study. As previously proposed ${ }^{(34)}$, the threshold effect of moderate adherence, but not higher adherence, to Fung and Mellen DASH indices might be due to some baseline psychological elements, such as neurotic or obsessive traits, present in some participants classified in the highest category of adherence to Fund and Mellen DASH indices. Although this might explain the attenuated effect of the Fung and Mellen DASH indices on depression risk in the highest category, this hypothesis could not be confirmed.

In order to verify that the definition of incident cases of depression (diagnosis of depression; diagnosis of depression and antidepressant treatment) did not affect the observed association in the present study, our analyses were repeated for both cases. Since both definitions of incident cases of depression showed similar results, the possibility of an important information bias due to depression misclassification does not seem very likely to affect our results. In any case, the potential misclassification of disease should be non-differential with respect to exposure in our prospective study, and it is well known that independent non-differential disease misclassification with very high specificity and low sensitivity does not bias the risk estimate ${ }^{(35)}$.

To our knowledge, only one previous randomized controlled trial has analysed the effect of a DASH diet on $\operatorname{mood}^{(36)}$. In that study, almost 100 postmenopausal women were assigned to follow a healthy diet or a DASH diet for 14 weeks and mood was measured by the Profile of Mood States. The authors found that the DASH diet appeared to improve mood to a greater extent than the healthy diet. However, that study reflects a short-term dietary intervention focused on postmenopausal women and the overall mood state, not depression.

The prospective design, data collection, data analysis and the inclusion of a large number of participants are notable strengths of our study. Our study also presented a high retention rate, the existence of published validation studies for our methods ${ }^{(15,21)}$ and a sufficiently large induction period, which reduces reverse causation bias. Moreover, we have used validated diagnoses of depression, adjusted for a wide array of potential confounders including personality traits, and excluded participants with other important diseases (such as diabetes and CVD) that may share some common pathophysiological mechanisms with depression. Moreover, it is not likely that reverse causality could have biased our results since cases reported in the first follow-up questionnaire were also excluded from the analyses.

Some limitations of our study should be acknowledged. First, a self-reported clinical diagnosis of depression was used and different subtypes or levels of depression were 
not taken into consideration. Second, the DASH diet indices were derived from a self-reported 136-item FFQ at baseline; however, previously published validation studies support the appropriateness of this methodology ${ }^{(15)}$. Changes in dietary intake during the follow-up period were not updated. Moreover, to date, there is not a perfect DASH index and the four indices used in the current study may present potential limitations previously mentioned in the discussion. These limitations may have introduced a non-differential misclassification bias that will lead to driving the crude estimate towards the null. However, previously published validation studies support the appropriateness of this methodology ${ }^{(15,21)}$.

\section{Conclusion}

In summary, the results obtained in the present study suggest that moderate adherence, but not higher adherence, to the Mellen and the Fung DASH diet indices might be associated with lower depression risk. Since these associations were non-linear, additional prospective studies are required to confirm these findings before the results can be generalized and clinical recommendations can be given.

\section{Acknowledgements}

Acknowledgements: The authors are indebted to the participants of the SUN Study for their continued cooperation and participation. They thank other members of the SUN Group: Alonso A., Benito S., de Irala J., de la Fuente-Arrillaga C., Delgado-Rodríguez M., Guillén- Grima F., Krafka J., Llorca J., López del Burgo C., Marti A., Martínez J.A., Pimenta A.M., Sánchez D., Serrano-Martínez M. and Vázquez Z. Financial support: This study was supported by the Spanish Ministry of Health and European Regional Development Fund (FEDER; grant numbers PI01/0619, PI030678, PI040233, PI042241, PI050976, PI070240, PI070312, PI081943, PI080819, PI1002658, PI1002293, RD06/0045, 2010/087 and G03/140), the Navarra Regional Government (grant numbers 45/2011 and 122/2014), and the University of Navarra. The funders had no role in the design, analysis or writing of this article. Conflict of interest: None declared. Authorship: The authors' contributions were as follows. M.A.M.-G., F.L.-R., A.S.-V. and P.M. designed the research; A.P.-C., M.A.M.-G. and M.B.-R. conducted the research; A.P.-C. and A.G. analysed the data and/or performed statistical analysis; A.P.-C. wrote the paper; A.S.-V. and P.M. provided essential materials; M.A.M.-G. and F.L.-R. were responsible for administrative support and funding; A.P.-C. and M.A.M.-G. had primary responsibility for the final content. All authors revised the manuscript for important intellectual content and read and approved the final manuscript. Etbics of buman subject participation: The Institutional
Review Board of the University of Navarra approved this study. Informed consent was implied by the voluntary completion of the first questionnaire.

\section{Supplementary material}

To view supplementary material for this article, please visit http://dx.doi.org/10.1017/S1368980016001531

\section{References}

1. Whiteford HA, Degenhardt L, Rehm J et al. (2013) Global burden of disease attributable to mental and substance use disorders: findings from the Global Burden of Disease Study 2010. Lancet 382, 1575-1586.

2. Sanchez-Villegas A \& Martinez-Gonzalez MA (2013) Diet, a new target to prevent depression? BMC Med 11, 3.

3. Perez-Cornago A, Lopez-Legarrea $\mathrm{P}$, de la Iglesia $\mathrm{R}$ et al. (2014) Longitudinal relationship of diet and oxidative stress with depressive symptoms in patients with metabolic syndrome after following a weight loss treatment: the RESMENA project. Clin Nutr 33, 1061-1067.

4. Jacobs DR Jr, Gross MD \& Tapsell LC (2009) Food synergy: an operational concept for understanding nutrition. $\mathrm{Am} \mathrm{J}$ Clin Nutr 89, issue 5, 1543S-1548S.

5. Sanchez-Villegas A, Delgado-Rodriguez M, Alonso A et al. (2009) Association of the Mediterranean dietary pattern with the incidence of depression: the Seguimiento Universidad de Navarra/University of Navarra follow-up (SUN) cohort. Arch Gen Psychiatry 66, 1090-1098.

6. Meng L, Chen DM, Yang Y et al. (2012) Depression increases the risk of hypertension incidence: a meta-analysis of prospective cohort studies. J Hypertens 30, 842-851.

7. Perez-Cornago A, de la Iglesia R, Lopez-Legarrea $\mathrm{P}$ et al. (2014) A decline in inflammation is associated with less depressive symptoms after a dietary intervention in metabolic syndrome patients: a longitudinal study. Nutr J 13, 36.

8. Hamer M \& Steptoe A (2012) Cortisol responses to mental stress and incident hypertension in healthy men and women. J Clin Endocrinol Metab 97, E29-E34.

9. Belmaker RH \& Agam G (2008) Major depressive disorder. $N$ Engl J Med 358, 55-68.

10. Dinh QN, Drummond GR, Sobey CG et al. (2014) Roles of inflammation, oxidative stress, and vascular dysfunction in hypertension. Biomed Res Int 2014, 406960.

11. Yannoutsos A, Levy BI, Safar ME et al. (2014) Pathophysiology of hypertension: interactions between macro and microvascular alterations through endothelial dysfunction. J Hypertens 32, 216-224.

12. Salehi-Abargouei A, Maghsoudi Z, Shirani F et al. (2013) Effects of Dietary Approaches to Stop Hypertension (DASH)-style diet on fatal or nonfatal cardiovascular diseases - incidence: a systematic review and meta-analysis on observational prospective studies. Nutrition 29, 611-618.

13. Domenech M, Roman P, Lapetra J et al. (2014) Mediterranean diet reduces 24-hour ambulatory blood pressure, blood glucose, and lipids: one-year randomized, clinical trial. Hypertension 64, 69-76.

14. Gea A, Bes-Rastrollo M, Toledo E et al. (2014) Mediterranean alcohol-drinking pattern and mortality in the SUN (Seguimiento Universidad de Navarra) Project: a prospective cohort study. Br J Nutr 111, 1871-1880.

15. de la Fuente-Arrillaga C, Ruiz ZV, Bes-Rastrollo M et al. (2010) Reproducibility of an FFQ validated in Spain. Public Health Nutr 13, 1364-1372.

16. Miller PE, Cross AJ, Subar AF et al. (2013) Comparison of 4 established DASH diet indexes: examining associations 
of index scores and colorectal cancer. Am J Clin Nutr 98, 794-803.

17. Guenther ALB, Liese AD, Bell RA et al. (2009) Association between the Dietary Approaches to Hypertension diet and hypertension in youth with diabetes mellitus. Hypertension 53, 6-12.

18. Dixon LB, Subar AF, Peters U et al. (2007) Adherence to the USDA food guide, DASH eating plan, and Mediterranean dietary pattern reduces risk of colorectal adenoma. $J$ Nutr 137, 2443-2450.

19. Fung TT, Hu FB, Wu K et al. (2010) The Mediterranean and Dietary Approaches to Stop Hypertension (DASH) diets and colorectal cancer. Am J Clin Nutr 92, 1429-1435.

20. Mellen PB, Gao SK, Vitolins MZ et al. (2008) Deteriorating dietary habits among adults with hypertension: DASH dietary accordance, NHANES 1988-1994 and 1999-2004. Arch Intern Med 168, 308-314.

21. Sanchez-Villegas A, Schlatter J, Ortuno F et al. (2008) Validity of a self-reported diagnosis of depression among participants in a cohort study using the Structured Clinical Interview for DSM-IV (SCID-I). BMC Psychiatry 8, 43 .

22. Martinez-Gonzalez MA, Lopez-Fontana C, Varo JJ et al. (2005) Validation of the Spanish version of the physical activity questionnaire used in the Nurses' Health Study and the Health Professionals' Follow-up Study. Public Health Nutr 8, 920-927.

23. Logan AC \& Jacka FN (2014) Nutritional psychiatry research: an emerging discipline and its intersection with global urbanization, environmental challenges and the evolutionary mismatch. J Physiol Anthropol 33, 22.

24. Baudrand R, Campino C, Carvajal CA et al. (2014) High sodium intake is associated with increased glucocorticoid production, insulin resistance and metabolic syndrome. Clin Endocrinol (Oxf) 80, 677-684.

25. Pan A, Keum N, Okereke OI et al. (2012) Bidirectional association between depression and metabolic syndrome a systematic review and meta-analysis of epidemiological studies. Diabetes Care 35, 1171-1180.

26. Perez-Cornago A, Zulet MA \& Martinez JA (2014) Association between mood and diet quality in subjects with metabolic syndrome participating in a behavioural weight-loss programme: a cross-sectional assessment. Nutr Neurosci 18, 137-144.

27. Payne ME, Steck SE, George RR et al. (2012) Fruit, vegetable, and antioxidant intakes are lower in older adults with depression. J Acad Nutr Diet 112, 2022-2027.

28. Tryon MS, Stanhope KL, Epel ES et al. (2015) Excessive sugar consumption may be a difficult habit to break: a view from the brain and body. J Clin Endocrinol Metab 100, 2239-2247.

29. Herbert J (2013) Cortisol and depression: three questions for psychiatry. Psychol Med 43, 449-469.

30. Martinez-Gonzalez MA \& Sanchez-Villegas A (2016) Food patterns and the prevention of depression. Proc Nutr Soc 75, 139-146.

31. Sanchez-Villegas A, Martinez-Gonzalez MA, Estruch R et al. (2013) Mediterranean dietary pattern and depression: the PREDIMED randomized trial. BMC Med 11, 208.

32. Sanhueza C, Ryan L \& Foxcroft DR (2013) Diet and the risk of unipolar depression in adults: systematic review of cohort studies. J Hum Nutr Diet 26, 56-70.

33. Jacka FN, Pasco JA, Williams LJ et al. (2013) Dietary intake of fish and PUFA, and clinical depressive and anxiety disorders in women. BrJ Nutr 109, 2059-2066.

34. Sanchez-Villegas A, Henriquez-Sanchez P, Ruiz-Canela M et al. (2015) A longitudinal analysis of diet quality scores and the risk of incident depression in the SUN Project. BMC Med 13, 197.

35. Rothman KJ, Greenland S \& Lash TL (editors) (2008) Modern Epidemiology. Philadelphia, PA: Lippincott Williams \& Wilkins.

36. Torres SJ \& Nowson CA (2012) A moderate-sodium DASHtype diet improves mood in postmenopausal women. Nutrition 28, 896-900. 